\title{
Monitoring of a CO Oxidation Reactor Through a Grey Model Based EKF Observer
}

\author{
by \\ Gianfranco Porru $\$$, Cosimo Aragonese\# and Roberto Baratti* \\ Dipartimento di Ingegneria Chimica e Materiali \\ Università degli Studi di Cagliari \\ Piazza D' Armi \\ I-09123 Cagliari \\ Italy \\ Email: baratti@dicm.unica.it
}

\begin{abstract}
Alberto Servida
Dipartimento di Chimica e Chimica Industriale

Università degli Studi di Genova

Via Dodecaneso, 31

I-16146 Genova

Italy

Email: servida@unige.it
\end{abstract}

* Author to whom the correspondence should be addressed

\$ Present address: SARTEC S.r.L., Traversa II Strada Est, Macchiareddu, C.P. 237, 09032 Assemini (Ca), Italy

\# On visit from CRS4, VI Strada Ovest Macchiareddu, I-09010 Uta (Ca), Italy 


\begin{abstract}
Often, in real applications it is difficult to dispose of a simple, yet, representative kinetic model because of the complexity of the reactions taking place. To overcome this limitation a hybrid modelling approach is proposed for the identification of the dynamic behaviour of chemical reactors. In particular, the tools of neural network modelling have been exploited to represent the kinetic reaction data. The "neural reaction rate model" is integrated within a first principles model that constitutes the basis of a nonlinear observer (Extended Kalman Filter, EKF) for an heterogeneous gas-solid reactor where the catalytic oxidation of carbon monoxide takes place. The outlined procedure shows that artificial neural networks (ANN) can be effectively used to formulate lumped reaction rates because of their capability in capturing the essential characteristics of the functional relationship among the state variables.
\end{abstract}




\section{Introduction}

The need for state estimators is particularly strong in chemical processes where, usually, the most important process variables are intrinsically difficult to measure. This has promoted the development of several observer applications where the critical primary process variables are estimated from secondary and more accessible ones. For instance, in polymerisation reactors the objective is to estimate the polymerisation degree, in biological reactors the prime aim is to evaluate the time evolution of the biomass composition, while in distillation columns and chemical reactors the critical problem concerns with the estimate of the outlet compositions of the relevant streams.

In a previous work (Baratti et al., 1993) it was shown that a good description of the reaction kinetics is critical for the development of robust and reliable reactor observers used to infer the outlet compositions of the main chemical species. This is not a trivial task since the kinetic analysis of complex reacting systems is a challenging problem. Even in presence of elementary reaction networks, the use of an unique global reaction rate law can be impossible because the rate determining steps may change by varying the operating conditions. A typical example is the carbon monoxide oxidation over Pt-alumina supported catalysts, where the rate determining step shifts from a surface reaction to an adsorption term depending on the temperature and carbon monoxide concentration (c.f., Herz and Marin, 1980; Goodman et al., 1982). For this system a conventional Langmuir-Hinshelwood rate law cannot describe the reaction kinetics over a wide range of operating conditions.

Often, detailed kinetic models are not suitable for on-line states/parameters estimation applications because of their numerical stiff characteristics. Thus, the development of concentration estimators must rely on the adoption of simple but representative lumped kinetic rate laws.

Starting from the earlier 90's the use of Artificial Neural Modelling (ANM) has been extensively exploited to describe chemical processes (c.f., Baht et al., 1990) because of their ability to approximate arbitrary complex functional relationships (Cybenko, 1989). Recently, ANM has been used to formulate approximate kinetic models for biological as well as conventional chemical reactors (c.f., Galvan et al., 1996; Thibault et al., 1997). Often the neural kinetic models are integrated within hybrid models (c.f., Thompson and Kramer, 1994), also called grey models, that have been proven to be effective for the development of reactor observers (c.f., Zorzetto and Wilson, 1996; Baratti and Servida, 1997).

In the present work, the use of first principles and neural modelling approaches is discussed for the development of a model-based estimator applied to a catalytic reactor where the catalytic oxidation of carbon monoxide over Pt-alumina supported catalysts takes place. This reaction was selected as test case since it exhibits, depending on the operating conditions, regions of extinction and ignition, and thus, it represents a reaction benchmark to evaluate the reliability and robustness of any adopted reactor model. 
The prime aim of this work is to assess the feasibility of using neural network modelling to represent lumped reaction rates capable to describe the steady-state behaviour of the catalytic reactor over the ignition and extinction regions. Indeed, for the oxidation of carbon monoxide, preliminary results have shown that it is not possible to use an unique LangmuirHinshelwood reaction rate expression to predict the performance of the catalytic reactor in both the regions of high and low reactivity (Aragonese, 1996).

The goal is to outline a procedure to integrate the reactor hybrid model within an observer to estimate the outlet reactor compositions. The observer, based on an Extended

Kalman Filter (EKF), is driven by the available on-line temperature measurements. Furthermore, the "neural reaction rate law" used in the dynamic reactor model is calibrated by simply using steady-state kinetic data.

The reliability of the proposed approach has been validated by comparison with experimental data acquired in a laboratory-scale reactor system.

\section{Experimental Apparatus}

The experimental apparatus consists of a catalytic tubular reactor (with recycle) equipped with an inlet flow control section, an analytical unit on the outlet stream, and a control section, as shown in the schematic layout reported in Figure 1.

Mass flow meters (Brooks, model 5850TR) are used to keep the carbon monoxide and the oxygen flowrates constant in time and to set the overall flow rate and composition of the feed stream to the desired specifications. The mass flow meters are operated through the control section.

The tubular reactor $(I D=0.012 \mathrm{~m} ; \mathrm{L}=0.356 \mathrm{~m})$ is jacketed with two resistances $(2 \mathrm{x}$ $360 \mathrm{~W}$ ) connected to a digital controller (independent loop). A layer of insulating material (0.03 $\mathrm{m}$ in thickness) is used to minimise heat losses. The reactor is packed with glass pellets to enhance heat transfer and turbulence. Only a thin layer $(0.01 \mathrm{~m})$ is packed with catalytic particles. In this way the conversion inside the tubular reactor is low enough to prevent significant temperature gradients along the reactor. The catalytic bed is equipped with a thermocouple located in the middle. The high flow rate avoids radial temperature gradients. The entering flow is preheated by a $320 \mathrm{~W}$ resistance to minimise longitudinal temperature gradient. The fluid exiting from the tubular reactor in cooled down to preserve the recirculation pump (Thomas, mod. 107-CD-18-TFEL-A). This is a membrane alternative pump equipped with an additional teflon membrane to maintain high purity, oil-free and dustfree gas. A stainless steel filter was inserted downstream to avoid, in the case of the pump failure, catalyst contamination. The reactor recirculation rate was set to an high value $(\mathrm{R}>$ 50) to make the reactor system to perform, from the fluid-dynamic point of view, as close as possible to a CSTR reactor. 
The reactor is equipped with a control unit constituted of a dedicated computer ( $\mu$ mac 5000 from Analog Device) connected to a PC that controlled the operating conditions and supervised the real-time acquisition of all the experimental data coming from the reactor and its analytical section.

The on-line analytical measurements were carried out through an IR analyser (LeyboldHeraeus BINOS mod. 4.1) connected to the reactor outlet. The on-line response was used to evaluate the estimator performance. The IR analyser signal was periodically checked off-line by GC analyses to verify its calibration.

The catalyst (platinum) is supported on $\gamma$-alumina (HARSHAW, type A1-0104T1/8, lot M-292) that provides an high surface-to-volume ratio and exhibits good mechanical properties. An egg-shell active site distribution was selected to reduce the platinum distribution density and to prevent the detrimental effects of diffusion phenomena. The catalyst was prepared by mono component impregnation as described elsewhere (Aragonese, 1996).

\section{The Mathematical Model}

To successfully develop a reliable and robust estimator, the adopted mathematical model has to be simple but capable to capture the main features of the plant dynamics. The model has to satisfy two conflicting requirements: the first is the on-line integrability, i.e. a simple enough model; the second is the need for the correct description of the functional relationship between state variables. The latter is critical because the estimation algorithm must rely on enough information to correctly infer the system state vector. In the following, we briefly illustrate the procedure that led to the formulation of a simple reactor model suitable for the integration within the estimator algorithm. A more detailed discussion on the identification of the concentration and temperature dynamics can be found in a previous work (Aragonese, 1996).

\subsection{Dynamics identification}

The dynamic behaviour of the reactor was investigated through a standard step response analysis performed in absence of reaction.

For the concentration dynamics, the reactor, operated at $433 \mathrm{~K}$, was initially flushed with a pure nitrogen stream $\left(0.037 \times 10^{-6} \mathrm{Nm}^{3} \mathrm{~s}^{-1}\right)$, then a step change in the inlet concentration was induced by adding a new stream of carbon dioxide. The results, shown in Figure $2 \mathrm{a}$, indicate that the overall fluid-dynamic of the system can be well described through a first order system with delay. Because the delay time, $32 \mathrm{~s}$, is due to the piping between the reactor and the on-line analyser, the reactor behaviour resembles that of a first-order system, 
and thus the concentration time evolution can be simply described through a conventional CSTR model:

$$
\tau_{c} \frac{d c}{d t}+c=b_{c} c_{i}
$$

where $\tau_{\mathrm{c}}$ (equal to $238.2 \mathrm{~s}$ ) is the characteristic time and $\mathrm{b}_{\mathrm{c}}=1$ is the gain constant.

To characterise the temperature dynamics, a temperature ramp was imposed because of the physical impossibility to produce an instantaneous temperature change. The system exhibited a response with overshoot, see Figure $2 b$, which is typical of interacting dynamic systems. Being our prime goal the formulation of a simple (but representative) dynamic model, the temperature time evolution has been approximated through a first order system:

$$
\tau_{\mathrm{T}} \frac{\mathrm{d} \mathrm{T}}{\mathrm{dt}}+\mathrm{T}=\mathrm{b}_{\mathrm{i}} \mathrm{T}_{\mathrm{i}}+\mathrm{b}_{\mathrm{w}} \mathrm{T}_{\mathrm{w}}+\mathrm{T}_{\text {pre }}
$$

where $\tau_{\mathrm{T}}(=48.5 \mathrm{~s})$ is the characteristic time, $\mathrm{b}_{\mathrm{i}}(=0.1738)$ and $\mathrm{b}_{\mathrm{w}}(=0.8262)$ are the gain constants with respect to the inlet and wall temperatures, respectively. The term $\mathrm{T}_{\text {pre }}$ accounts, in an effective fashion, for: a) the heat exchanges that take place in the preheater and in the cooling systems ; b) the heat dispersion occurring through the piping (not thermally insulated). These contributions have been lumped in an effective parametric model through the use of the term $\mathrm{T}_{\text {pre }}$, in the attempt to keep the dynamic model of the system as simple as possible. Indeed, the approach has proved to be able to capture the essential dynamic features of the systems as the identification run reported in Figure $2 b$ shows. In actuality, the results indicate that even though the simple model well recovers the time response of the system it doesn't describe properly the interactions between the reactor itself and the auxiliary equipment (preheater, cooling system, and connecting piping). This is the reason why the model is not capable to predict the overshoot in the reactor temperature as shown in Figure $2 b$ that, indeed, refers to a critical test in which the wall temperature was forced to change of about $30^{\circ} \mathrm{C}$ over a ramp lasting only $3 \mathrm{~min}$.

\subsection{The reaction kinetic model}

The neural kinetic model was calibrated making use of steady-state kinetic data. These were obtained through a set of experiments carried out at three different temperatures for various values of the inlet CO concentration. The results are shown in Figure 3, where the observed reaction rate, $\mathrm{R}_{\mathrm{obs}}$, is plotted as a function of the bulk $\mathrm{CO}$ concentration for the three temperatures.

As mentioned in the Introduction, ANM has been applied to formulate a lumped kinetic reaction rate for the oxidation of carbon monoxide over Pt-alumina supported catalyst. As pointed out in previous studies (c.f., Goodman et al., 1982; Aragonese, 1996), the development of classical models is particularly hard because of the strong non-linearity of the 
functional relationship between state variables (carbon monoxide bulk concentration and bulk temperature) and the reaction rate, and because of the shift in the rate determining step. Our intent is to develop an unique kinetic expression, valid over the entire region of the input space of interest, suitable for the integration within an observer structure.

An Artificial Neural Network (ANN) is a mathematical structure consisting of independent computational elements called nodes or neurones. Each neurone performs a weighted sum of its inputs and transforms the sum in the output signal through an activation function. In a Multilayered Feed Forward Artificial Neural Network (MFFANN) neurones are arranged in a layered structure. Neurones take inputs from every node of the previous layer and produce an output signal that serves as input to the nodes of the next layer. There is a one-way flow of information: from the input to the output nodes. The input and hidden layers are augmented with an extra neurone, the bias, that provides a constant output signal equal to one. A MFFANN has been used, and the common sigmoidal activation function has been adopted for the neurones of the hidden and of output layers:

$$
f(x)=\frac{1}{1+e^{-x}}
$$

Many studies have demonstrated the ability of ANN's to represent complex kinetic data (Galvan et al., 1996; Kito et al., 1994; Sasaki et al., 1995). Neural network modelling is usually considered a black box approach, since, in principle, no a priori knowledge of the process is needed. It is sufficient to provide the network with the set of input data (bulk carbon monoxide concentration and bulk temperature) and the corresponding output outcomes (reaction rate, corresponding to the input values): the network calculates its own outputs from the given set of input data and, by a training algorithm, the weights of the connections between neurones are modified so as to minimise the difference between the desired output outcomes and the calculated ones. During the training procedure, the neural network learns the relationship between the inputs and the outputs, builds an internal model, and then, given a new set of input data, can calculate the corresponding unknown outputs. In the present work the training was driven using the Osborn's modification of LevenbergMarquardt algorithm.

Our goal is not a mere optimal fit of the steady-state experimental data, but the development of a neural kinetic model that captures, as well as possible, the essential characteristics of the functional relationships between inputs (concentration and temperature) and outputs (reaction rate). In other words, the neural kinetic model must also provide consistent derivatives of the reaction rate with respect to the concentration and temperature. This additional requirement has been imposed because the kinetic model is part of an hybrid model whose Jacobian matrix is used to estimate the prediction error covariance within the observer structure. The observer can apply right corrections only if the Jacobian is correctly computed, that is, the trend predicted by the neural kinetic model must be as close as possible 
to the actual one. To achieve this result, we have tested different MFFANN structures with different numbers of hidden layers and neurones. Various network architectures have been trained, always maintaining the same pair of input and output data. Every time we tested the extrapolation capabilities of the neural model into input space regions different from those used for the training. The consistency test on the derivatives of the reaction rate with respect to the bulk concentration and temperature has been used as additional criterion to assess the "goodness" of the neural kinetic model.

The results of this analysis led to select a MFFANN with two nodes (+ bias) in the input layer, four nodes (+ bias) in the hidden layer, and one node in the output layer.

Because large changes in the input values can cause numerical problems (Psichogis and Ungar, 1992), we have used, as inputs to the network, dimensionless carbon monoxide bulk concentration: $\mathrm{x}_{1}=\mathrm{C}_{\mathrm{CO}} / \mathrm{C}_{\mathrm{r}}$ and dimensionless bulk temperature: $\mathrm{x}_{2}=2\left(\mathrm{~T}-\mathrm{T}_{\mathrm{av}}\right) / \Delta \mathrm{T}_{\max }$. With these scaling laws, the temperature ranged between -1 and 1 , while the $\mathrm{CO}$ concentration varied between 0 and 3 . This configuration has proven to provide the best results.

Since the network's output ranges from 0 to +1 , we have defined a dimensionless reaction rate as follows:

$$
\mathrm{R}_{\mathrm{ANN}}=\frac{\mathrm{R}_{\mathrm{obs}}}{\mathrm{c}_{\mathrm{r}}} \mathrm{f}_{\mathrm{s}} .
$$

The scaling factor, $\mathrm{f}_{\mathrm{S}}$, is necessary because the dimensionless reaction rate varies from $10^{-6}$ to $10^{-5}$ and using a sigmoidal function it is impossible to appreciate the differences between them.

Figure 3 shows the measured and estimated reaction rate at three different temperatures. As we can see, the reaction rate prediction is rather good in both the ignition and extinction regions. It is a remarkable result considering that the reaction rate undergoes changes of over one order of magnitude in the transition from the ignition to the extinction zone, and that, contrary to conventional kinetic models, an unique simple neural kinetic model is capable to describe the complete set of experimental data.

\subsection{The reactor mathematical model}

The adopted reactor model is constituted of a simplified reactor description augmented with a "neural reaction rate". The reactor model relies on the following assumptions: a) the reacting system is a well mixed tank reactor, b) negligible intraphase mass and heat transport resistances, c) negligible accumulation of: reactants and products within the catalyst pellets and of heat within the gaseous mixture, d) all the physicochemical properties do not depend on temperature and composition. The relevant model equations are:

$$
\frac{\mathrm{d} \mathrm{u}}{\mathrm{dt}}=\theta\left(\mathrm{v}_{\mathrm{i}} \mathrm{u}_{\mathrm{i}}-\mathrm{v} \mathrm{u}\right)-\mathfrak{R}(\mathrm{u}, \mathrm{v})
$$




$$
\frac{\mathrm{d} v}{\mathrm{~d} t}=\theta \kappa\left(\mathrm{v}_{\mathrm{i}}-\mathrm{v}\right)-\delta\left(\mathrm{v}-\mathrm{v}_{\mathrm{w}}\right)+\omega \mathfrak{R}(\mathrm{u}, \mathrm{v})+\mathrm{Q}_{\text {pre }}
$$

with I.C's

$$
\mathrm{u}(0)=\mathrm{u}_{0} ; \mathrm{v}(0)=\mathrm{v}_{0}
$$

where:

$$
\begin{gathered}
u=\frac{C}{C_{r}} ; v=\frac{T}{T_{r}} \\
\theta=\frac{q}{V} ; \omega=\frac{(-\Delta H) c_{r}}{\rho C p T_{r}} ; \delta=\frac{U S}{\rho C p V} ; \kappa=\frac{\rho_{g} C p_{g}}{\rho C p}
\end{gathered}
$$

$\mathrm{R}(\mathrm{c}, \mathrm{T})$ is the modified neural reaction rate, $\mathrm{q}$ is the volumetric flow rate evaluated at the reference temperature $T_{r}(=433 \mathrm{~K}), \mathrm{V}$ is the reactor volume, $u_{i}$ and $v_{i}$ represent the dimensionless $\mathrm{CO}$ concentration and temperature of the feed stream, respectively. The above dimensionless parameters are related to $\tau_{\mathrm{T}}, \tau_{\mathrm{c}}, \mathrm{b}_{\mathrm{i}}$ and $\mathrm{b}_{\mathrm{w}}$ through the relationships reported in a previous paper (Baratti et al., 1993).

A more detailed discussion on $\mathrm{Q}_{\text {pre }}$ is needed. As mentioned in the previous section $\mathrm{Q}_{\text {pre }}$ accounts for the net heat contribution given by the piping, preheater and cooling system. Now, being the reactor, from a heat point of view, an interactive system this contribution will change depending if we operate the reactor in absence on in presence of reaction. To simplify the analysis, the description of all the experimental runs has been done by adopting the average value of $\mathrm{Q}_{\text {pre }}$ calculated from the data corresponding to the reactor operated in the ignition region. Even though this value (equal to $1.45 \times 10^{-4} \mathrm{~s}^{-1}$ ) is significantly different from that calculated in absence of reaction (equal to $8.58 \times 10^{-4} \mathrm{~s}^{-1}$ ) a reasonable description of all the experimental runs has been achieved.

\section{Extended Kalman Filter}

The extended Kalman filter was developed on the basis of the approximate reactor model defined by eqs. (3) - (4); the procedure is extensively discussed in the previous paper by Baratti et al.(1993). Here, it is enough to outline the basic concepts on which EKF relies, while for the details on the governing EKF equations we refer to Gelb (1974, pg. 188). The extended Kalman filter is an approximate optimal estimator that infers the vector state variable, $\mathbf{x}$, at a given time, on the basis of the predictions of an approximate process model and of secondary measurements available up to that time. In short, the inferential procedure developed within the Kalman frame is as follows: 


$$
\mathbf{x}_{\mathrm{k}+1 / \mathrm{k}+1}=\mathbf{x}_{\mathrm{k}+1 / \mathrm{k}}+\mathbf{K}_{\mathrm{k}+1}\left(\mathbf{z}_{\mathrm{k}+1}-\mathbf{h}_{\mathrm{k}+1}\right)
$$

where $\mathbf{x}_{\mathrm{k}+1 / \mathrm{k}+1}$ represents the estimated vector of state variables at the time step $(\mathrm{k}+1), \mathbf{x}_{\mathrm{k}+1 / \mathrm{k}}$ is the prediction vector of the approximate dynamic process model, $\mathbf{z}_{\mathrm{k}+1}$ is the vector of the secondary measurements, $\mathbf{h}_{\mathrm{k}+1 / \mathrm{k}}$ is the vector of the observed state variables as predicted by the process model, and $\mathbf{K}_{\mathrm{k}+1}$ is the filter gain matrix that weights the relative importance given to the model predictions and to the actual measurements. The filter gain matrix is constructed on the basis of the error covariance matrices of the measurements (r) and of the $\operatorname{model}(\mathbf{Q})$.

Actually, the entries of the matrices $\mathbf{Q}$ and $\mathbf{r}$ can be regarded as the tuning parameters of the Kalman filter and have been computed through a minimum least-square criterion, where the function to be minimised was the square of the deviations between the estimated and the measured values of the product composition i.e., the outlet carbon dioxide composition.

It is worth to point out that the only real-time dynamic information supplied to the EKF is the actual reactor bulk temperature, while the neural kinetic model only provides the steady-state reaction rate at the actual conditions.

\section{Results and Discussion}

The performance of the nonlinear observer was evaluated, for different inlet $\mathrm{CO}$ concentrations, by performing transient kinetic experiments driven by externally forcing the reactor wall temperature.

In a previous work (Baratti et al., 1993), it was shown that the numerical method adopted to integrate the EKF equations is not critical, thus, a simple Euler method has been used. The integration time, equal to the sampling time, has been set equal to $6 \mathrm{~s}$.

As previously discussed, the entries of the error covariance matrices, $\mathbf{Q}$ and $\mathbf{r}$, are regarded as tuning parameters and have been evaluated once for all by using the data of a reference run. In the reference run, the wall temperature (dashed line) was changed externally as shown in Figure $4 \mathrm{a}$, while the total flow rate $\left(0.037 \times 10^{-6} \mathrm{Nm}^{3} \mathrm{~s}^{-1}\right)$ and the inlet CO mole fraction (0.011) were kept constant. In Figure $4 \mathrm{~b}$, the predicted estimates (dashed line) are compared with the actual measurements (continuous line) in terms of the outlet $\mathrm{CO}_{2}$ mole fraction as a function of time. The computed values of the error covariance matrices have been kept constant for all the successive experimental runs.

In the first experiment, the reactor wall temperature (dashed line) was forced as shown in Figure $5 \mathrm{a}$ where the reactor temperature (continuous line) is also reported. The inlet flow rate $\left(0.037 \times 10^{-6} \mathrm{Nm}^{3} \mathrm{~s}^{-1}\right)$ and the inlet $\mathrm{CO}$ mole fraction (0.012) were kept constant throughout 
the entire experiment. The selected operating conditions made the reactor to operate in the ignited regime.

To better illustrate the advantage of using an estimator, the performance of the model alone was assessed by simply using eqs. (3)-(5). The comparison between the experimental results (continuous line) and the predictions (dotted line) is shown in Figure 5b, in terms of the outlet $\mathrm{CO}_{2}$ mole fraction. The agreement is only qualitative and this is due to the inaccuracy of the approximate model. In the same Figure the prediction of the outlet $\mathrm{CO}_{2}$ mole fraction obtained with the EKF (dashed line) is also shown. The improvement is clear, and the use of the observer allowed to achieve an average error of $2 \%$ with a maximum error of $5 \%$. It is worth pointing out that in this experiment the interaction phenomena seem to play a minor role since no overshoot in the reactor temperature is observed. This is also the reason why the simplified hybrid model well recovers the experimental wall temperature.

The second experiment has been carried out in the spent zone in order to test the feasibility of using the same neural kinetic model, the same covariance matrices and parameters adopted for the ignition zone. The prescribed wall temperature profile (dashed line) and the corresponding reactor temperatures (continuous line) are reported in Figure 6a. The inlet carbon monoxide mole fraction (0.03) and the total flow-rate $\left(0.037 \times 10^{-6} \mathrm{Nm}^{3} \mathrm{~s}^{-1}\right)$ were kept constant.

Let us examine the results obtained by only integrating the reactor model. In the extinction region, the predictions of the temperature (dotted line in Figure 6a) and of the outlet $\mathrm{CO}_{2}$ mole fraction (dotted line in Figure $6 \mathrm{~b}$ ) appear to be poorer than those obtained in the ignition region. This could be due to the fact that for $\mathrm{Q}_{\text {pre }}$ the average value corresponding to the ignition zone has been selected, and this, as previously discussed, is significantly lower than that calculated in absence of reaction. Decreasing the value of Qpre the model predicts a lower reactor temperature, and consequently, the predicted outlet $\mathrm{CO}_{2}$ mole fraction decreases.

When the estimator is used to compute the outlet $\mathrm{CO}_{2}$ mole fraction the agreement is improved as shown in Figure 6b, where the estimated mole fraction (dashed line) is reported as a function of time.

The results show that neural modelling can provide a useful framework to represent lumped reaction rate laws, and that it is viable to pursue this strategy by examining more complex applications.

\section{Conclusions}

The feasibility of using grey models, based on first principles and neural modelling approaches, has been discussed and demonstrated for the case of a catalytic reactor where the catalytic oxidation of carbon monoxide over Pt-alumina supported catalysts takes place. The 
reaction case study has also provided the opportunity to outline a generalized procedure for the development and integration of hybrid modelling within a Kalman filter.

The use of Artificial Neural Networks has proven to be very effective in describing the kinetics of a reacting system that exhibits ignition and extinction phenomena. Furthermore, while the neural kinetic model was capable to describe the system kinetics over the entire range of investigated operating conditions, a conventional Langmuir-Hinshelwood rate law failed to provide a correct representation.

The formulated estimator based on hybrid modelling well describes the reactor dynamics in both the ignition and extinction regions, even though, the neural reaction rate model was developed on the basis of steady-state data.

\section{Acknowledgement}

The work has been partially supported through an EC contribution (BRRT-CT97-5023). 


\section{Notation}

$b_{c} \quad$ concentration gain constant

$b_{i} \quad$ inlet temperature gain constant

$\mathrm{b}_{\mathrm{w}} \quad$ wall temperature gain constant

c concentration, $\mathrm{mol} \mathrm{m}^{-3}$

$c_{r} \quad$ reference concentration $\left(=0.281 \mathrm{~mol} \mathrm{~m}^{-3}\right)$

$\mathrm{Cp} \quad$ specific heat capacity, $\mathrm{J} \mathrm{kg}^{-1} \mathrm{~K}^{-1}$

$\mathrm{f}_{\mathrm{S}} \quad$ scaling factor $\left(=10^{-5}\right)$

K Kalman filter gain matrix

q volumetric flow rate evaluated at $\mathrm{T}=\mathrm{T}_{\mathrm{r}}, \mathrm{m}^{3} \mathrm{~s}^{-1}$

$\mathrm{Q}_{\text {pre }} \quad$ net heat $=\mathrm{T}_{\text {pre }} / \mathrm{T}_{\mathrm{r}}(\theta \kappa+\delta)=1.45410^{-4} \mathrm{~s}^{-1}$

$\mathrm{R}=\mathrm{R}_{\mathrm{ANN}} / \mathrm{f}_{\mathrm{S}} / \mathrm{V}$

$\mathrm{R}_{\text {ANN }}$ neural reaction rate, $\mathrm{m}^{3} \mathrm{~s}^{-1}$

$\mathrm{R}_{\mathrm{obs}} \quad$ neural reaction rate, $\mathrm{mol} \mathrm{s}^{-1}$

$\mathrm{S} \quad$ heat exchange surface, $\mathrm{m}^{2}$

$\mathrm{t} \quad$ time, $\mathrm{s}$

$\mathrm{T}$ temperature, $\mathrm{K}$

$\mathrm{T}_{\mathrm{r}} \quad$ reference temperature $(=433 \mathrm{~K})$

$\mathrm{T}_{\text {pre }} \quad$ net temperature increases, $\mathrm{K}$

$\mathrm{u}$ dimensionless concentration, $\mathrm{c} / \mathrm{c}_{\mathrm{r}}$

$\mathrm{U} \quad$ heat transfer coefficient, $\mathrm{J} \mathrm{m}^{-2} \mathrm{~s}^{-1} \mathrm{~K}^{-1}$

$\mathrm{V}$ dimensionless temperature, $\mathrm{T} / \mathrm{T} \mathrm{r}$

$\mathrm{V} \quad$ estimated reactor volume $\left(=1.18210^{-3} \mathrm{~m}^{3}\right)$

$\mathbf{x}$ state vector

$\mathbf{z}$ measurement vector

Greek letters

$\delta \quad=\mathrm{U} \mathrm{S} / \rho \mathrm{Cp} \mathrm{V}=0.017 \mathrm{~s}^{-1}$

$\Delta \mathrm{H} \quad$ reaction heat $=282000 \mathrm{~J} \mathrm{~mol}^{-1}$

$\theta \quad=\mathrm{q} / \mathrm{V}=4.18910^{-3} \mathrm{~s}^{-1}$

$\kappa \quad=\rho_{\mathrm{g}} \mathrm{Cp} \mathrm{p}_{\mathrm{g}} / \rho \mathrm{Cp}=0.880$

$\rho \quad$ density, $\mathrm{kg} \mathrm{m}^{-3}$

$\omega=(-\Delta H) c_{r} / \rho C p T_{r}=149.434 \mathrm{~s}^{-1}$

\section{Subscript}

$\begin{array}{ll}\text { av } & \text { average value } \\ \text { i } & \text { reactor inlet } \\ \text { g } & \text { gas state } \\ \text { w } & \text { wall temperature }\end{array}$




\section{References}

Aragonese, C., 1996 "Sviluppo del Filtro di Kalman Esteso per un Reattore Catalitico con Molteplici Reazioni, Ignizioni e Fenomeni di Isteresi", Ph. D. Dissertation, University of Bologna, Italy.

Baht, N. V., Minderman, P. A., McAvoy, T. J. and Wang, N. S., 1990, Modelling Chemical Process Systems via Neural Computation, IEEE Control Sys. Mag., 10, 24.

Baratti, R., J. Alvarez and M. Morbidelli, 1993, Design and Experimental Verification of a Nonlinear Catalytic Reactor Estimator, Chem. Eng. Sci., 48, 2573.

Baratti, R. and Servida, A., 1997, Development of a Nonlinear Observer Based on Neural and First Principles Modelling, Neural Networks in Engineering Systems, EANN'97, (A.B. Bulsari and S. Kaillo Eds), 135.

Cybenko, G., 1989, Approximation by Superpositions of a Sigmoidal Function, Math. Control, Signals Sys., 2, 303.

Galvan, K. I., Zaldivar, J. M., Hernandez, H., Molga, E., 1996, The Use of Neural Networks for Fitting Complex Kinetic Data, Computers Chem. Engng, 20, 1451.

Gelb, A., 1974, Applied Optimal Estimation, M.I.T. Press, Cambridge.

Goodman, M. G., Cutlip, M. B., Kenney, C. N., Morton, W. and Mukesh, D., 1982, Transient Studies of Carbon Monoxide Oxidation over Platinum Catalyst, Surface Sci., 120, L453.

Herz, R. K. and Marin, S. P., 1980, Surface Chemistry Model of Carbon Monoxide Oxidation on Supported Platinum Catalysts, J. Catal., 65, 281.

Kito, S., Hattori, T. and Murakami, Y., 1994, Estimation of Catalytic Performance by Neural Network - Product Distribution in Oxidative Dehydrogenation of Ethylbenzene, Applied Catalysis A: General, 114, L173.

Psichogios, D. C. and Hungar, L. H., 1992, A Hybrid Neural Network - First Principles Approach to Process Modelling, AIChE J., 38, p 1499.

Sasaki, M., Hamada, H., Kintaichi, Y. and Ito, T., 1995, Application of a Neural Network to the Analysis of Catalytic Reactions. Analysis of NO Decomposition over Cu/ZSM-5 Zeolite, Applied catalysis A: General, 132 , 261.

Thibault, J., AcuZa Leiva, G. and Grandjean, B. P. A., 1997, "Identification of Kinetic Rate Expression in Fermentation Systems", Neural Networks in Engineering Systems, EANN'97, (A.B. Bulsari and S. Kaillo Eds), 123.

Thompson, M.L. and Kramer, M.A., 1994, Modeling Chemical Processes Using Prior Knowledge and Neural Networks, AIChE J., 40, p 1328.

Zorzetto, L. F. M. and Wilson, J. A., 1996, "Monitoring Bioprocesses Using Hybrid Models and an Extended Kalman Filter", Computers Chem. Engng, 20, S689. 


\section{Captions for Figures}

Figure 1. Sketch of the experimental apparatus.

Figure 2. (a) Response to a step change in the inlet $\mathrm{CO}_{2}$ concentration equal to $0.83 \%$ (flow rate equal to $0.037 \times 10^{-6} \mathrm{Nm}^{3} \mathrm{~s}^{-1}$; reactor temperature equal to $423 \mathrm{~K}$. (b) Reactor temperature response to a change in the wall temperature as a function of time (flow rate equal to $0.037 \times 10^{-6} \mathrm{Nm}^{3} \mathrm{~s}^{-1}$ ).

Figure 3. Reaction rate as a function of carbon monoxide bulk concentration at three different temperatures: 423,433 and $443 \mathrm{~K}$.

Figure 4. Observer performance (reference run): (a) wall (dashed line) and reactor (continuous line) temperature as a function of time; (b) comparison between experimental (continuous line) and estimated (dashed line) outlet $\mathrm{CO}_{2}$ mole fraction. Inlet $\mathrm{CO}$ fraction equal to 0.011 .

Figure 5. Observer performance (first run): (a) wall (dashed line), model (dotted line) and reactor (continuous line) temperature as a function of time; (b) comparison between experimental (continuous line), model (dotted line) and estimated (dashed line) outlet $\mathrm{CO}_{2}$ mole fraction. Inlet $\mathrm{CO}$ fraction equal to 0.012 .

Figure 6. Observer performance (second run): (a) wall (dashed line), model (dotted line) and reactor (continuous line) temperature as a function of time; (b) comparison between experimental (continuous line), model (dotted line) and estimated (dashed line) outlet $\mathrm{CO}_{2}$ mole fraction. Inlet $\mathrm{CO}$ fraction equal to 0.03 . 


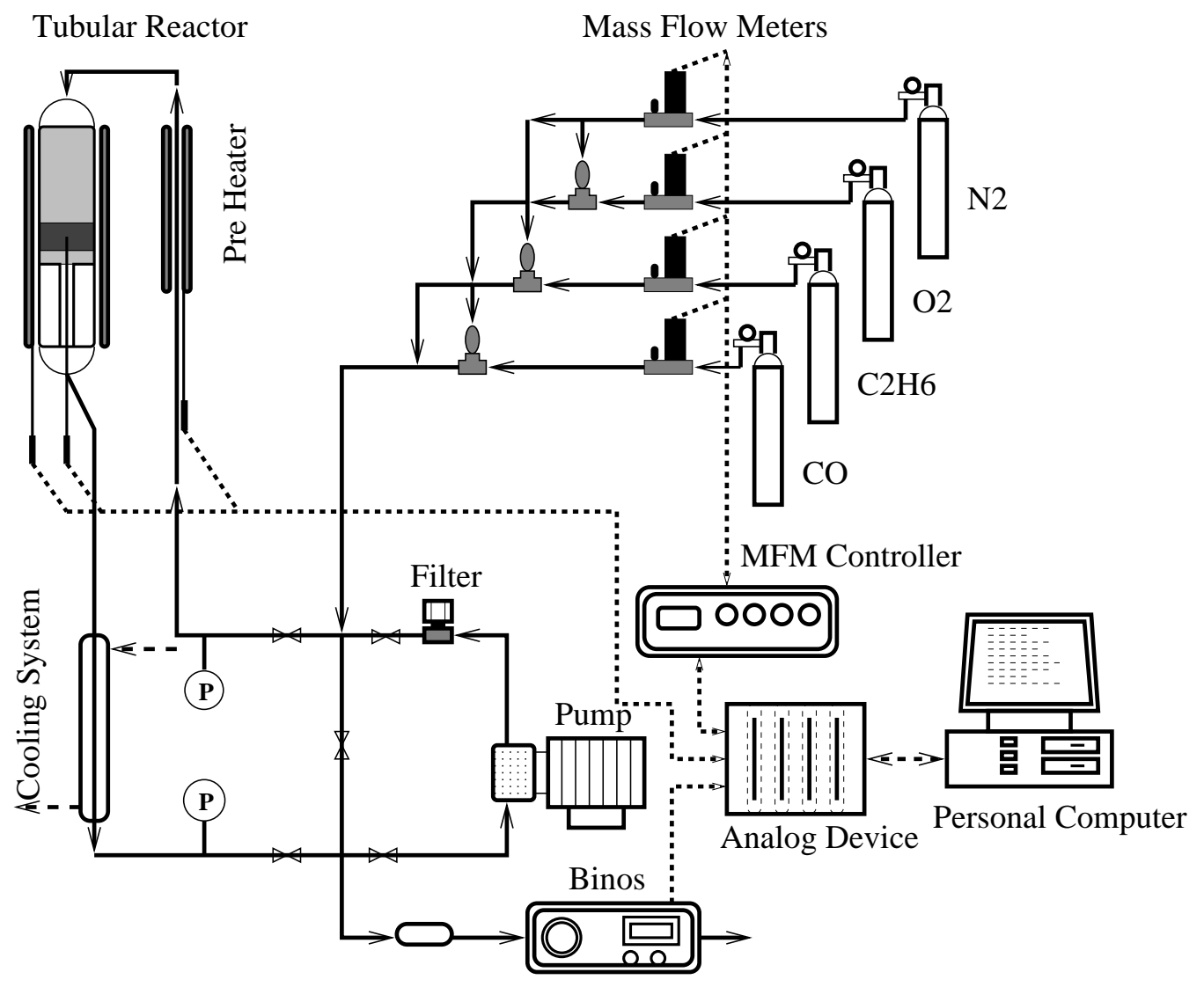

Figure 1 
Figure 2

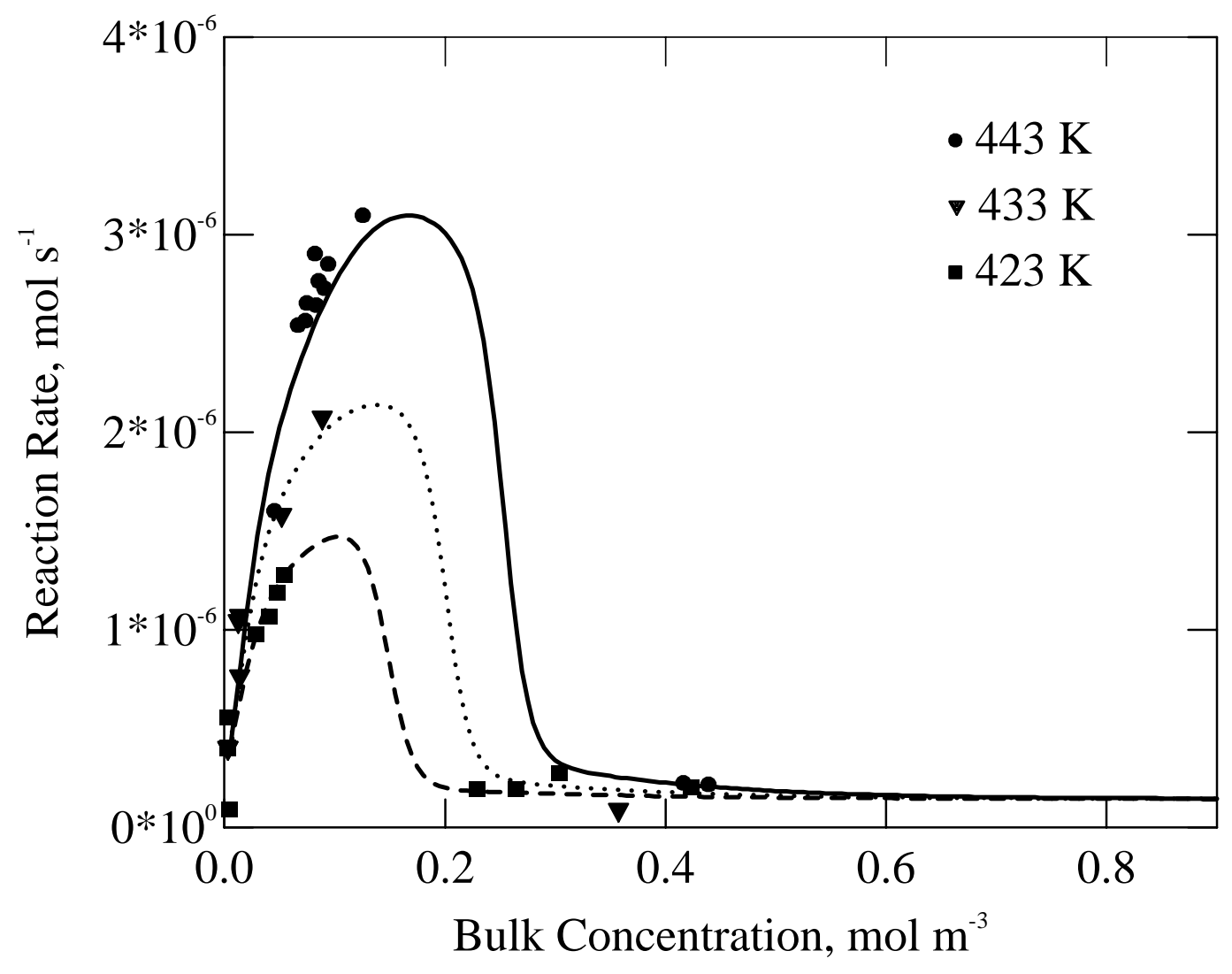


Figure 3
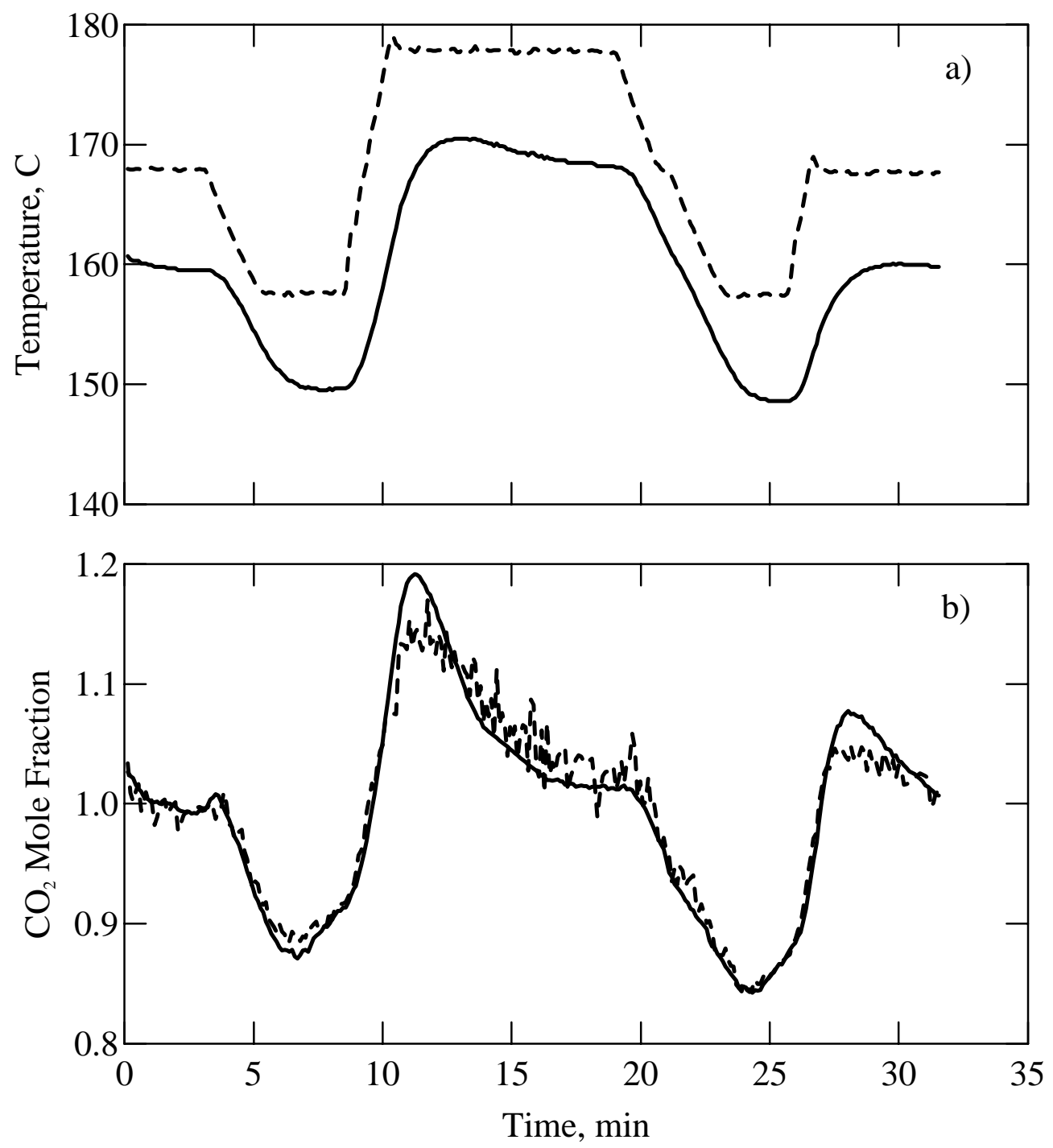
Figure 4
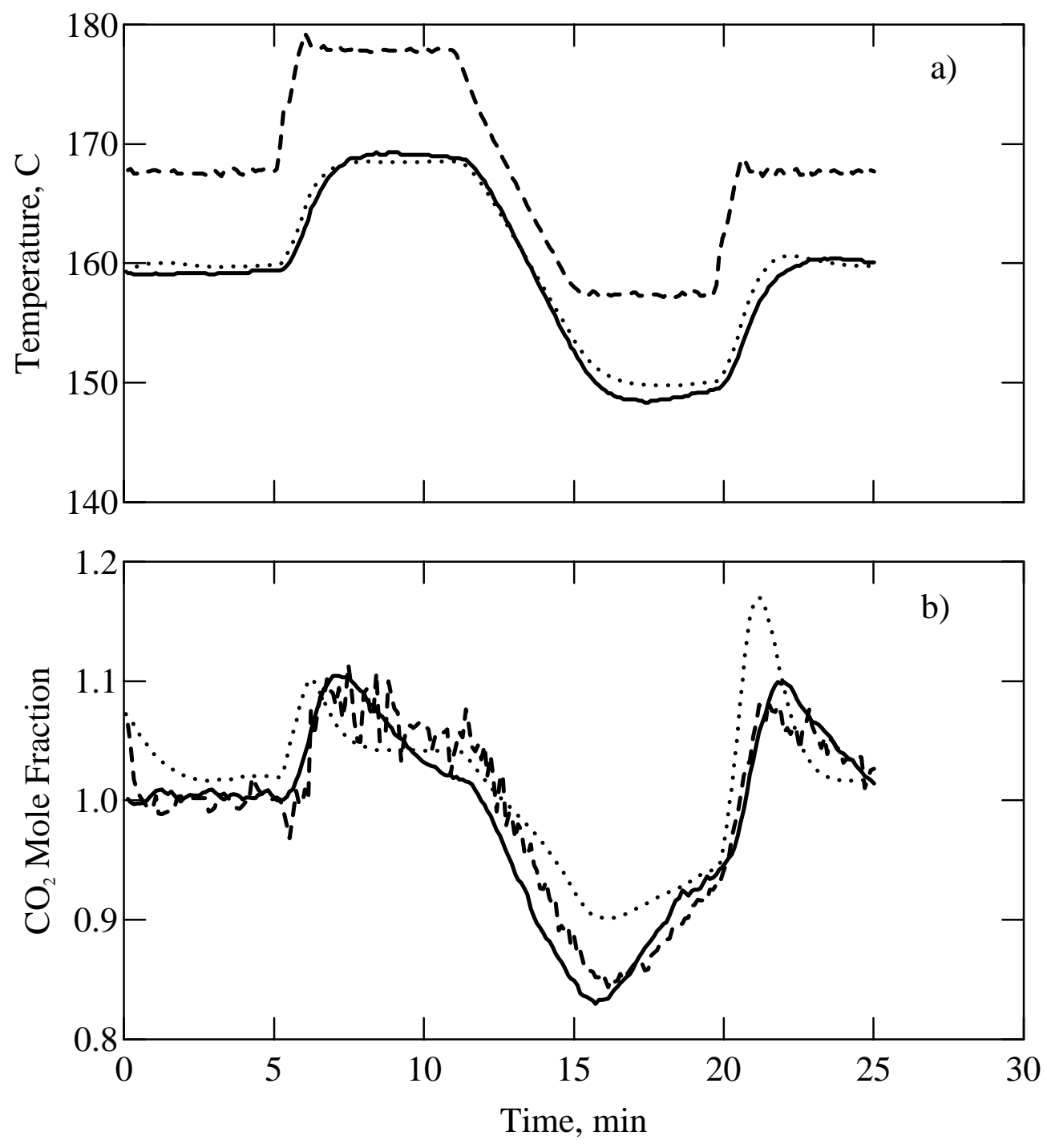
Figure 5
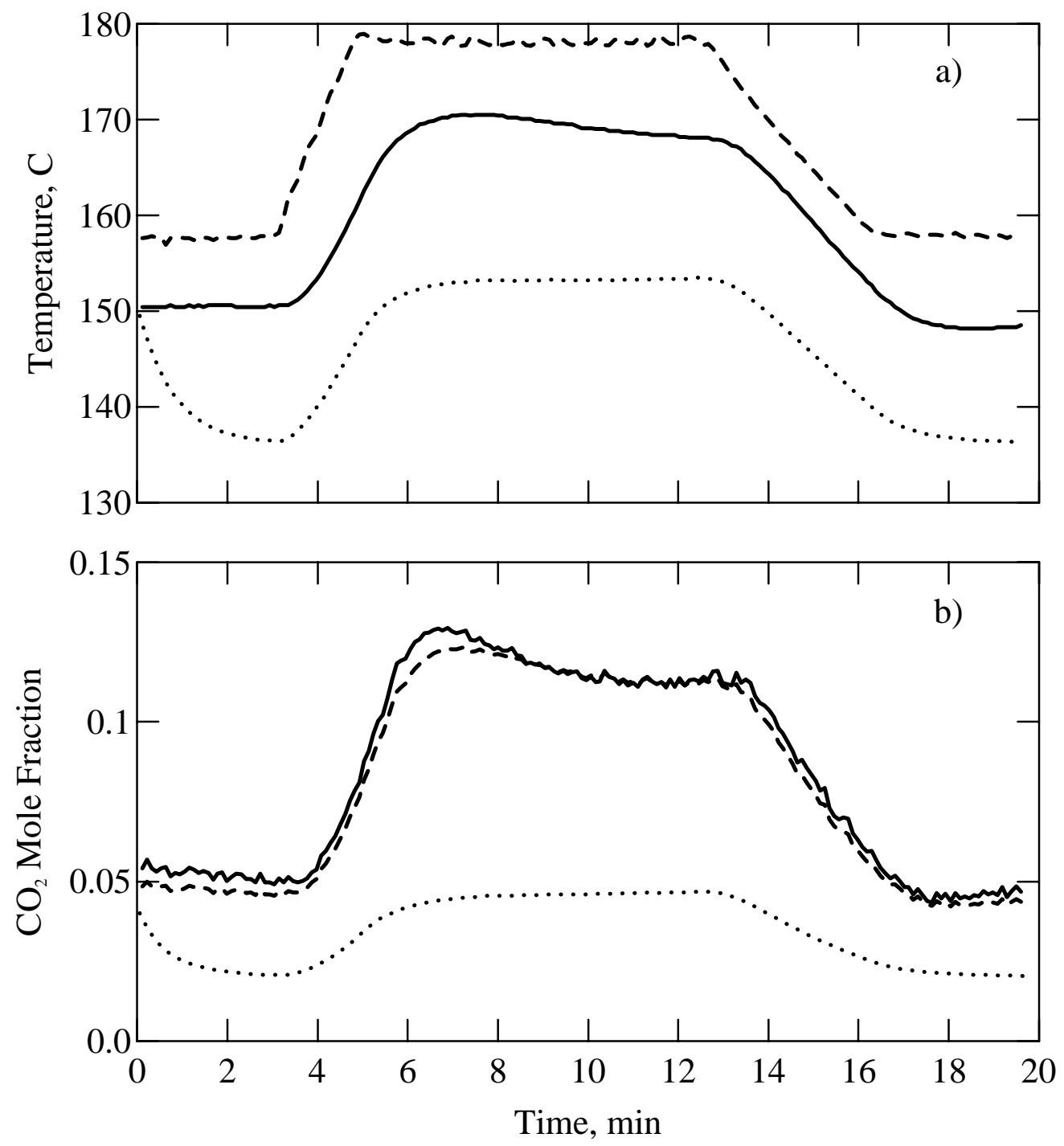